\section{Orale Immuntherapie bei Erdnussallergie erfolgreich}

Erdnussallergien sind potenziell lebensgefährlich. Da es für betroffene Kinder im Vereinigten Königreich keine krankheitsmodifizierenden Interventionsmöglichkeiten gibt, haben britische Forscher den Erfolg einer oralen Toleranzinduktion überprüft.

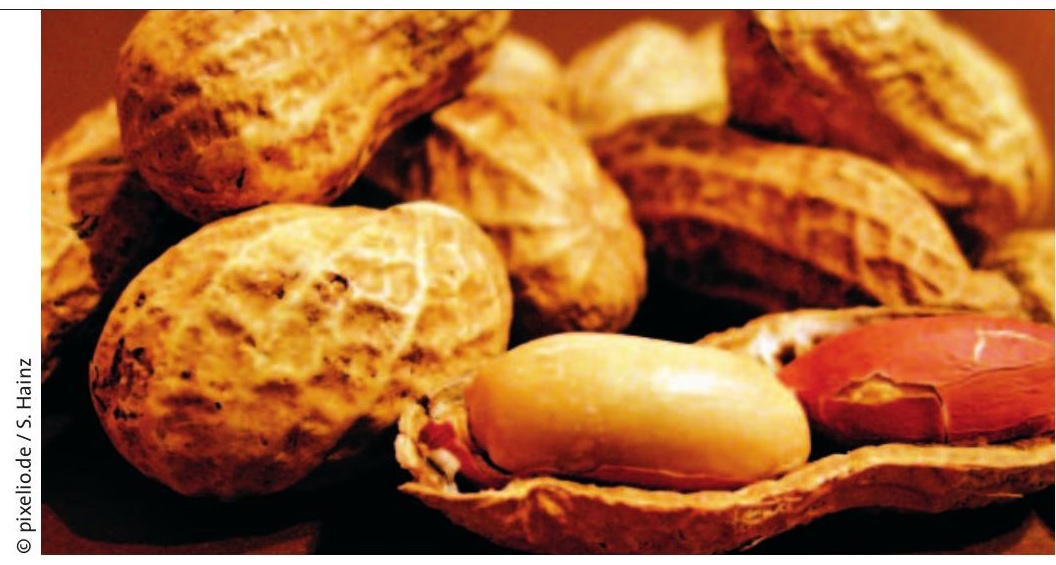

$\mathrm{D}$ ie Lebensqualität von Kindern mit Erdnussallergie und deren Familien ist aufgrund der ständigen Angst vor Fehlern beim Essen und einer drohenden Anaphylaxie stark beeinträchtigt. Da es bislang keine kausale Therapie gibt, untersuchte eine britische Arbeitsgruppe bei vier betroffenen 9- bis 13-Jährigen die Wirksamkeit einer oralen Immuntherapie. Die Wissenschaftler bestätigten die Erdnussallergie zunächst unter anderem mittels doppelblinder, placebokontrollierter oraler Provokation und erfassten hierbei die Erdnussprotein-Dosis, ab der eine allergische Reaktion eintrat. Der Grenzwert lag zwischen $5 \mathrm{mg}$ und $50 \mathrm{mg}$ (ein Vierzigstel bis ein Viertel einer Erdnuss).
Während der oralen Immuntherapie nahmen die Kinder einmal täglich mit Joghurt vermischtes Erdnussmehl zu sich. Die Initialdosis basierte auf dem individuellen Erdnussprotein-Grenzwert und der klinischen Schwere der Symptome. Alle zwei Wochen wurde die Dosis gesteigert, bis die Kinder täglich 800 mg Erdnussprotein zu sich nahmen. Nach sechs Wochen unter dieser Erhaltungsdosis führten die Autoren eine weitere orale Provokation durch, um den Grenzwert neu zu ermitteln. Jetzt konnten alle Kinder zehn Erdnüsse tolerieren (etwa 2,38 g Erdnussprotein). Ihre Grenzwerte waren etwa um das 48-, 49-, 55- beziehungsweise 478 -fache gestiegen.

\title{
Nur leichte Stichanaphylaxie: hyposensibilisieren oder nicht?
}

Bei Patienten mit systemischen Reaktionen auf Bienen- oder Wespenstiche verbessert die spezifische Immuntherapie die Lebensqualität. Ob das auch für Patienten gilt, die nur auf der Haut Überempfindlichkeitsreaktionen haben, wurde in einer kleinen offenen Studie untersucht.

In Europa wird die spezifische Immuntherapie (SIT) vor allem bei Patienten angewandt, die nach Bienen- oder Wespenstichen systemische Reaktionen entwickeln. Unter der Therapie berichten die Betroffenen über eine wieder deutlich ansteigende Lebensqualität - im Gegensatz zu denjenigen, die ausschließlich einen Notfall-Adrenalin-Injektor erhalten.

An einer offenen Studie nahmen 55 Patienten teil, die gegen Wespengift sensibilisiert waren und bislang nur Hautreaktionen auf Stiche gezeigt hatten. Sie wurden über die Behandlungsmöglichkeiten und das relativ geringe Risiko für eine zukünftige systemische Reaktion nach Insektenstichen aufgeklärt. 29 der Patienten wurden randomisiert einer SIT zugewiesen oder erhielten nur einen Adrenalin-Injektor. 26 weitere Studienteilnehmer wählten ihre Behandlung aktiv aus (11 SIT, 15 Adrenalin-Injektor). Während des Beobachtungszeitraums wurde die Einschränkung der Lebensqualität durch die Therapie mithilfe des Vespid Allergy Quality of Life Questionnaire (VQLQ) dokumentiert.

Die Lebensqualität verbesserte sich unter der Hyposensibilisierung hochsignifikant gegenüber der Injektor-Gruppe, in
Fazit: Bei allen Kindern - inklusive einem mit anaphylaktischer Reaktion bei der initialen oralen Provokation - konnte der Erdnussprotein-Grenzwert durch die orale Immuntherapie deutlich erhöht werden. Die Intervention war erfolgreich, sodass schließlich zehn Erdnüsse toleriert werden konnten. Die Autoren weisen jedoch darauf hin, dass ihre Ergebnisse vorläufig sind und die orale Erdnuss-Provokation derzeit noch nicht außerhalb von Studien erfolgen sollte.

pe

Clark AT. Successful oral tolerance induction in severe peanut allergy.

Allergy. 2009;64:1218-20 der die VQLQ bei vielen Allergikern sogar absank. Die Patienten aus der SITGruppe empfanden ihre Behandlung nicht als belastend und stuften diese Behandlung gegenüber dem ständigen Mitführen eines Adrenalin-Injektor als überlegen ein. 83\% der Injektor-Patienten wollten nach Abschluss der Studie ebenfalls eine SIT beginnen.

Fazit: Patienten mit isolierten Hautreaktionen nach Insektenstichen stufen ihre krankheitsbezogene Lebensqualität unter einer Hyposensibilisierung viel besser ein als Patienten, die mit einem AdrenalinAutoinjektor versorgt werden.

bk

Oude Elberink JNG et al. Immunotherapy improves health-related quality of life of adult patients with dermal reactions following yellow jacket stings.

Clin Exp Allergy 2009; 39: 883-9 